\title{
SIMULAÇÃO DE PROCESSOS DE TRANSFERÊNCIA DE CALOR APLICADA À INDÚSTRIA ALIMENTÍCIA COM ENFOQUE EM PRODUTOS CÁRNEOS
}

\section{SIMULATION OF HEAT TRANSFER PROCESSES APPLIED TO THE FOOD INDUSTRY WITH FOCUS ON MEAT PRODUCTS}

\author{
I. V. C. TORRES ${ }^{1, *}$, T. C. SOUZA ${ }^{2}$, A. M.O., SIQUEIRA ${ }^{3}$
}

1 Universidade Federal de Viçosa, Departamento de Química; Viçosa/MG, Brasil; E-mail: isadora.torres@ufv.br

2 Universidade Federal de Viçosa, Departamento de Química; Viçosa/MG, Brasil; E-mail: tainara.souza@ufv.br

3 Universidade Federal de Viçosa, Departamento de Química; Viçosa/MG, Brasil; E-mail: antonio.siqueira@ufv.br; ORCID: https://orcid.org/0000-0001-9334-0394

\begin{tabular}{|c|}
\hline A R T I C LE INFO \\
\hline $\begin{array}{l}\text { Article history: } \\
\text { Received 2020-02-22 } \\
\text { Accepted 2020-07-15 } \\
\text { Available online 2020-12-12 }\end{array}$ \\
\hline $\begin{array}{l}\text { palavras-chave } \\
\text { Carne bovina } \\
\text { Transferência de calor } \\
\text { Simulação de processos }\end{array}$ \\
\hline $\begin{array}{l}\text { keywords} \\
\text { Beef } \\
\text { Heat transfer } \\
\text { Process simulation }\end{array}$ \\
\hline
\end{tabular}

\begin{abstract}
R E S U M O
O objetivo desse artigo é o estudo dos processos de transferência de calor em produtos cárneos. A metodologia adotada foi o fracionamento da carne do tipo "coxão mole", a construção de uma malha igualmente espaçada nas coordenadas x e y e a aferição das temperaturas nas regiões nodais em diferentes intervalos de tempo. A partir da temperatura calculada no tempo zero, pode-se prever as temperaturas nos demais tempos, utilizando o método explícito das diferenças finitas e, assim, foi possível comparar os resultados obtidos com as temperaturas experimentais. Os erros encontrados entre os dados experimentais e os calculados foi da faixa de oitenta por cento, distorção que pode ser justificada pelos erros associados ao experimento. Ademais, por meio da aferição das temperaturas centrais podese estudar a cocção da carne e classificá-las como mal passadas, ao ponto ou bem passadas. Conclui-se que o experimento obteve êxito no estudo do método das diferenças finitas bem como na análise da importância dos fenômenos de transferência de calor na indústria alimentícia.
\end{abstract}




\section{INTRODUÇ̃̃̃O}

O curso de Engenharia Química da Universidade Federal de Viçosa (UFV) tem como parte da sua matriz curricular a disciplina de Fenômenos de Transporte II, em que vários aspectos de transferência de calor são evidenciados. Mas, é importante deixar claro alguns conceitos para que o entendimento seja completo. Assim, o calor pode ser definido como a forma de energia que pode ser transferida de um sistema para outro em consequência da diferença de temperatura entre eles e a transferência de calor é a ciência responsável por esse estudo (ÇENGEL; GHAJAR, 2012).

A Equação 1 é a equação que descreve a transferência de calor de forma geral e para encontrar sua solução serão feitas algumas considerações e também algumas simplificações ao longo do trabalho (INCROPERA; DEWITT, 2014).

$$
\frac{\partial}{\partial x}\left(k \frac{\partial T}{\partial x}\right)+\frac{\partial}{\partial y}\left(k \frac{\partial T}{\partial y}\right)+\frac{\partial}{\partial z}\left(k \frac{\partial T}{\partial z}\right)+\dot{q}=\rho \cdot c \cdot \frac{\partial T}{\partial t}
$$

Nesse caso, $k$ é a condutividade térmica, $\rho$ é a massa específica do material, $c$ é a capacidade calorífica do material, $\dot{q}$ é a taxa de geração de energia, $\frac{\partial T}{\partial t}$ é a parcela do regime transiente e são levadas em consideração as variações de temperatura ao longo das três coordenadas espaciais: $x, y$ e $z$, já que a equação explicitada diz respeito às coordenadas retangulares. Os procedimentos para o cálculo poderão variar entre complexidade e precisão (INCROPERA; DEWITT, 2014).

No que faz referência às aplicações da transferência de calor, têm-se que elas são infinitas, podendo ser destacadas (BEJAN, 1996):

- Isolamento (por fibra de vidro) de tetos e paredes de edifícios para manter determinadas condições climáticas;

- Quantificação da perda de energia através de janelas modernas e isoladas para manter o ambiente confortável tanto no inverno quanto no verão;

- Projeto e operação de geradores de vapor (caldeiras) ou ebulidores requer a compreensão da transferência de calor que ocorre da queima (combustão) de carvão, gás ou óleo para a água nos tubos;

- Dissipação de calor em linhas de potência elétrica devido à resistência elétrica;

- Processos sanitários, manuseio de lixo, esterilização;

- Manuseio e processamento de alimentos.

O último tópico será a aplicação evidenciada neste estudo, justamente por acreditar que nos dias atuais os hábitos dos brasileiros têm sido redefinidos a partir da consolidação da indústria alimentícia, existindo por um lado, uma preocupação com a qualidade e composição dos alimentos a serem consumidos e por outro lado, uma necessidade de rapidez do preparo, devido exigências da sociedade contemporânea, que cada vez têm menos tempo para atividades corriqueiras (FRANÇA et al., 2012).

Nesse sentido, o objeto de estudo escolhido foi a carne bovina, justamente por ser um componente central da dieta humana, tanto como alimento direto quanto como ingrediente essencial a vários outros produtos. Vale ressaltar que, devido à sua importância, é natural que a carne atraia controvérsias, especialmente nos dias atuais, em que a busca por produtos "saudáveis" é prerrogativa de um consumidor que se sente informado, mas que, muitas vezes, segue um modismo ditado pela mídia e desconhece os reais benefícios e riscos decorrentes do seu consumo (RAMOS; GOMIDE, 2017).

Vale ressaltar que a carne deve ser cozida até uma temperatura interna ideal, que irá depender do grau de cozimento que se pretende alcançar. $\mathrm{O}$ cozimento provoca a desnaturação da proteína globina do pigmento da mioglobina, expondo o grupo heme e o tornando mais propenso à oxidação e à consequente formação do pigmento globina hemicromo, de cor marrom-castanha característica. O calor irá afetar a coloração interna da carne, que passará de vermelho-brilhante característico quando cru para um vermelho-claro, e em seguida, para marrom-acinzentado quando a temperatura interna aumenta de $50{ }^{\circ} \mathrm{C}$ para $80^{\circ} \mathrm{C}$ (RAMOS; GOMIDE, 2017).

Temperaturas internas entre $71{ }^{\circ} \mathrm{C}$ e $72{ }^{\circ} \mathrm{C}$ são consideradas ideais para o cozimento da carne, pois eliminam os principais patógenos presentes, incluindo Listeria monocytogenes, Salmonella e Escherichia coli. Entretanto, pode ocorrer um fenômeno de escurecimento prematuro devido a desnaturação da mioglobina - e, consequentemente, uma aparência cozida, em uma temperatura mais baixa que o normal, ou seja, a carne pode aparecer totalmente cozida apesar de não ter atingido a temperatura interna segura, indicada para eliminar patógenos (RAMOS; GOMIDE, 2017). Assim, há um reconhecimento que o uso de termômetros no dia a dia é pouco usual e, então, acredita-se que explorar e expor insumos que possam ajudar os consumidores no momento de preparação da carne torna-se de extrema relevância.

Dessa forma, o desenvolvimento do trabalho consiste na avaliação do tempo de aquecimento necessário para que a carne bovina "coxão mole" esteja pronta, de acordo com os diferentes estágios de preparo: mal passado, ao ponto e bem passado, utilizando conceitos da transferência de calor, bem como suas metodologias de cálculo, mais precisamente as equações de Diferenças Finitas pelo Método Explícito. O intuito é enriquecer conhecimentos sobre produtos cárneos e potencializar processos da indústria alimentícia.

\subsection{Fundamentação Teórica de Grupamentos Adimensionais}

Quando uma equação é descrita ou desenvolvida, ela precisa apresentar uma homogeneidade dimensional, ou seja, as equações que envolvem grandezas físicas devem ter as mesmas unidades de medida de ambos os lados do sinal de igualdade (PACÍFICO, 2017).

Outro tópico que pode ser abordado é o da análise dimensional. Vale deixar claro que ela só tem sentido quando o número de variáveis adimensionais para descrever o fenômeno físico for menor que o número de variáveis físicas dimensionais envolvidas no fenômeno igualdade (PACÍFICO, 2017). Assim sendo, existem diversos grupamentos adimensionais que são associados com o intuito de descrever parâmetros de protótipos que podem ser utilizados em modelos e nesse estudo estarão descritos alguns dos números adimensionais necessários para a descrição do sistema. 
O primeiro número a ser analisado será o número de Nusselt $(\mathrm{Nu})$ que representa uma relação entre a transferência de calor por convecção e a condução de calor do mesmo fluido:

$$
N u=\frac{h \cdot L}{k}
$$

em que, $\mathrm{k}$ é a condutividade térmica do ar, [W/(m.K)], L corresponde ao comprimento característico do corpo, definido pela razão entre a área e o perímetro do sólido, [m.]. As correlações recomendadas para o número de Nusselt são (INCROPERA; DEWITT, 2014):

- Superfície superior da placa aquecida ou superfície inferior da placa resfriada:

$$
\begin{array}{rc}
\overline{N u}_{L}=1 & R a_{L} \leq 10^{4} \\
\overline{N u}_{L}=0,54 R a_{L}{ }^{1 / 4} & 10^{4} \leq R a_{L} \leq 10^{7} \\
\overline{N u}_{L}=0,15 R a_{L}{ }^{1 / 3} & 10^{7} \leq R a_{L} \leq 10^{11}
\end{array}
$$

- Superfície inferior da placa aquecida ou superfície superior da placa resfriada:

$$
\overline{N u}_{L}=0,27 R a_{L}^{1 / 4} \quad 10^{5} \leq R a_{L} \leq 10^{10}
$$

Sequencialmente, outro número que é importante para a modelagem do sistema é o número de Rayleigh, que descreve um balanço entre as forças que promovem a convecção e as forças que se opõem a esse fenômeno (FISCHER, 2017), matematicamente, tem-se a Equação 3:

$$
R a=\frac{g \cdot \beta \cdot\left(T_{S}-T_{\infty}\right) \cdot L^{3}}{v \cdot \alpha}
$$

Onde $g$ é a aceleração da gravidade, $\beta$ é o coeficiente de expansão volumétrica, nesse caso dado como $1 / T_{f}$, em que $T_{f}$ é a temperatura de película, $\Delta T$ é a diferença de temperatura entre a carne e a superfície da churrasqueira, $L$ é o espaçamento entre as placas, $v$ é a viscosidade cinemática e $\alpha$ é a difusividade térmica (FISCHER, 2017).

Outra forma de se calcular o número de Rayleigh é relacionando com os números adimensionais de Grashof $\left(G r_{L}\right)$ e Prandtl $(\mathrm{Pr})$. O número de Prandtl aproxima a viscosidade cinemática e a difusividade térmica de um fluido, expressando a relação entre a difusão de quantidade de movimento e a difusão da quantidade de calor do próprio fluido. O número de Grashof é a relação entre a sustentação de um fluido e sua viscosidade dinâmica $(\mu)$ (FISCHER, 2017).

\section{ANÁLISE E SIMULAÇÃO DO PROCESSO}

\subsection{Considerações Efetuadas}

Algumas considerações são necessárias para que se possa distender o processo de cálculo da transferência de calor da carne bovina. Desta maneira, assume-se que todas as medidas de temperatura da rede nodal, em cada instante, foram feitas ao mesmo tempo; o regime adotado é o transiente; a transferência de calor é bidimensional, ocorrendo apenas no eixo $x$ e no eixo $y$; os efeitos de geração de energia através da resistência térmica serão computados por meio do termo $\dot{q}$ da equação 1 , em que por considerações envolvendo uma superfície muito fina, haverá uma condução direta da taxa volumétrica de calor gerada pela churrasqueira Grill para a amostra de carne disposta sobre ela. $\mathrm{O}$ valor de $\dot{q}$ calculado levando-se em conta a taxa de geração volumétrica da churrasqueira. A Figura 1 representa as dimensões da churrasqueira utilizada.

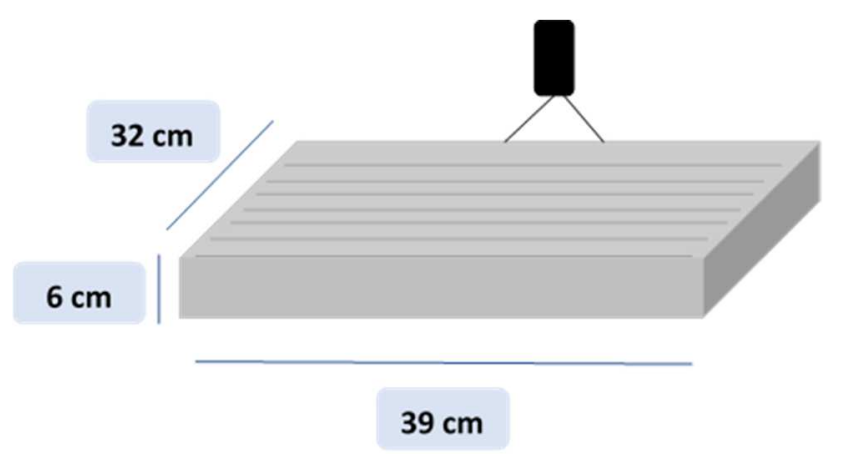
Figura 1 - Croqui representativo das dimensões da churrasqueira. Fonte: Autores.

Nesse sentido, foi avaliado a potência efetiva associada à churrasqueira. Por meio das informações do fabricante, tem-se que à potência da churrasqueira é de $1250 \mathrm{~W}$, porém, é esperado que a potência efetiva transferida à carne seja menor, devido às perdas para o ambiente, o que implica na redução da sua eficiência. No que diz respeito a esse tópico supracitado, foi utilizada a referência de Demarchi, et al. (2012) e segundo dados obtidos, tem-se que para uma churrasqueira de $1800 \mathrm{~W}$ a eficiência mínima é em torno de $14 \%$. Realizando os cálculos de proporção para a potência da churrasqueira utilizada no experimento, assim sendo:

$$
\frac{1250 W}{1800 W} \cdot 14 \%=9,72 \%
$$

Realizando-se, então, a aproximação de eficiência para $10 \%$, tem-se, portanto:

$$
\begin{gathered}
\text { Pot }_{\text {Efetiva }}=\operatorname{Pot}_{\text {Real }} \cdot \eta \\
\text { Pot }_{\text {Efetiva }}=1250 \mathrm{~W} \cdot 0,10 \\
\text { Pot }_{\text {Efetiva }}=125 \mathrm{~W}
\end{gathered}
$$


Como hipótese, considerou-se que a taxa de geração foi obtida a partir da transferência da potência efetiva pelo volume da churrasqueira. Dessa forma, obtêm-se:

$$
\begin{gathered}
\dot{q}=\frac{\text { Pot }_{\text {Efetiva }}}{\text { Volume }_{\text {Churrasqueira }}} \\
\dot{q}=\frac{125 \mathrm{~W}}{(0,390 \cdot 0,320 \cdot 0,06) \mathrm{m}^{3}} \\
\dot{q}=1,6693,4 \frac{\mathrm{W}}{\mathrm{m}^{3}}
\end{gathered}
$$

Assim, utilizou-se o valor de $1,6710^{4} \mathrm{~W} / \mathrm{m}^{3}$ como taxa de geração volumétrica de calor, para todos os cálculos subsequentes.

Além disso, o processo ocorre apenas com mudanças físicas (sem nenhuma reação química); as propriedades termofísicas da carne não variam e por fim, assume-se que a geometria da carne utilizada no experimento é a de uma placa plana e, portanto, utiliza-se coordenadas cartesianas para fins de cálculo. A Figura 3 apresenta uma amostra de carne utilizada no experimento, juntamente, com a rede nodal elaborada para a medição dos pontos.

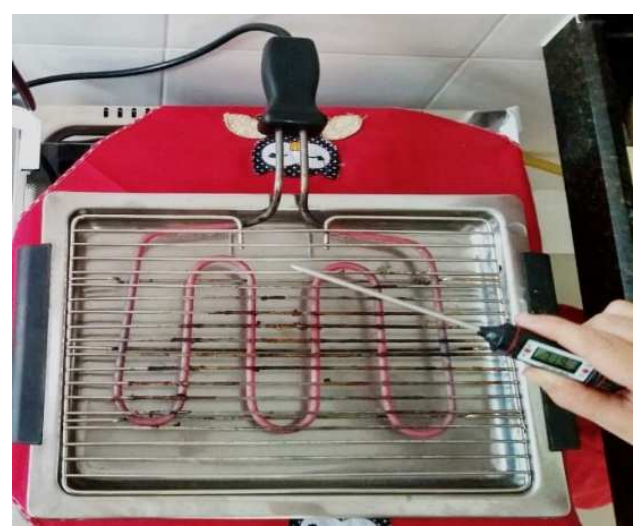

Figura 2 - Churrasqueira Grill utilizada no experimento. Fonte: Autores.

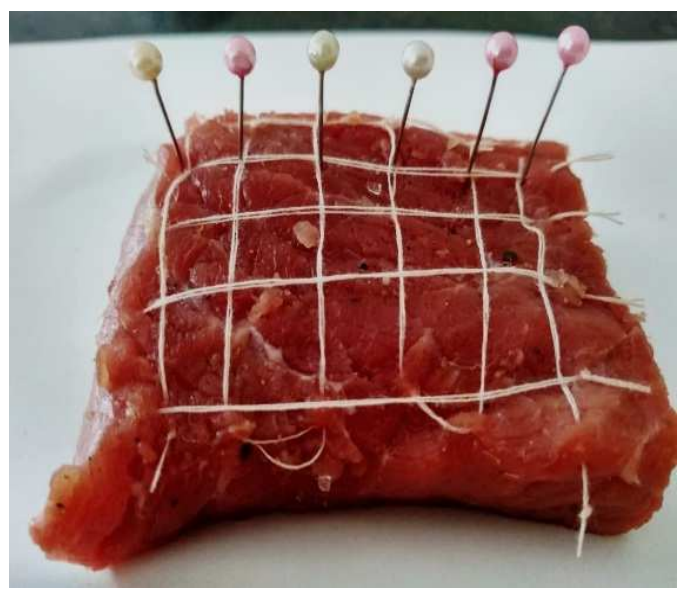

Figura 3 - Amostra de carne utilizada no experimento com a confecção da rede nodal. Fonte: Autores.

\subsection{Metodologia Experimental}

O procedimento experimental iniciou-se fracionando a carne do tipo "coxão mole" em cortes homogêneos de 5 centímetros de largura, 6 centímetros de comprimento e 2,5 centímetros de espessura, conforme Figura 4.

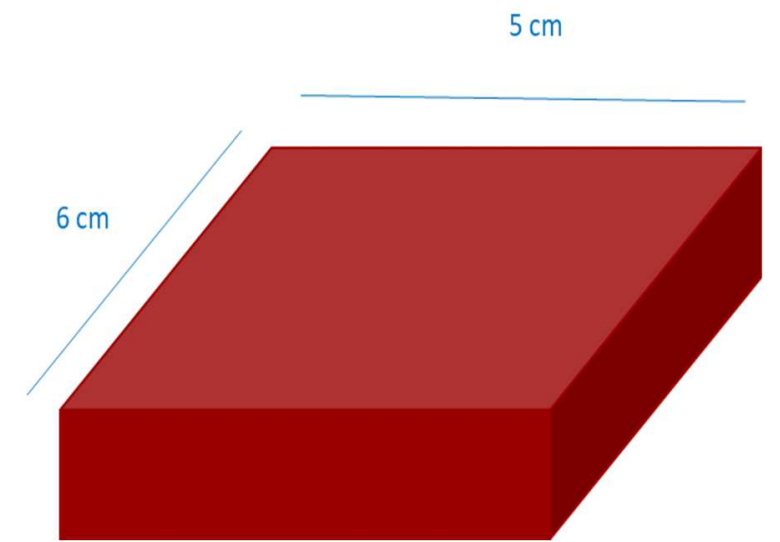

Figura 4 - Croqui representativo do fracionamento da carne "coxão mole".

Fonte: Autores.

Ressalta-se que a escolha do tipo de carne "coxão mole" deve-se a grande utilização desse corte na culinária, visto às suas fibras curtas e à sua maciez (RAMOS; GOMIDE, 2017).

Posteriormente, foi costurado uma malha refinada no corte bovino, igualmente espaçada no eixo x e no eixo $\mathrm{y}$, com um distanciamento de $1 \mathrm{em} 1 \mathrm{~cm}$, como representado na Figura 5.

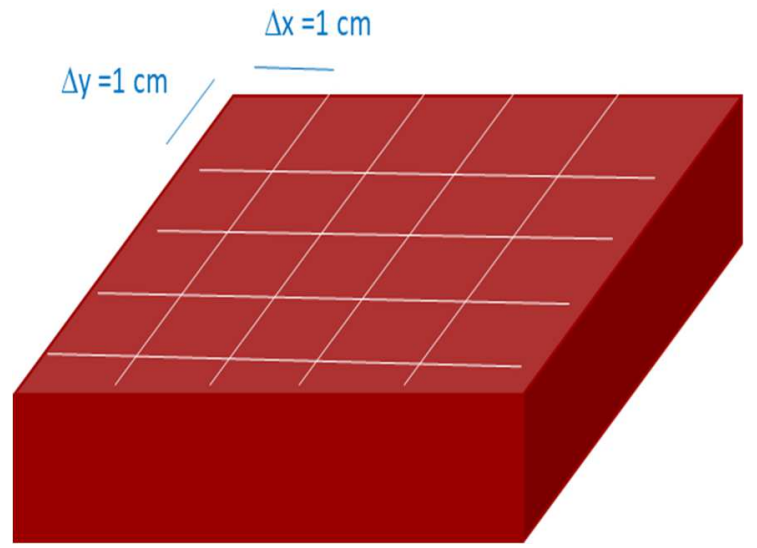

Figura 5 - Croqui representativo da construção da malha na fração de "coxão mole". Fonte: Autores.

Foi necessária a aferição da temperatura da carne, em cada um dos pontos nodais, previamente ao início do experimento. Em seguida, ligou-se a churrasqueira elétrica, deixando-a em repouso até que a temperatura de sua grelha estivesse aproximadamente constante. Ademais, foi aferida a temperatura ambiente e a temperatura do fluido acima da churrasqueira, utilizando um termômetro digital.

Colocou-se, então, a fração de carne sobre a churrasqueira e, a cada intervalo de 5 minutos, foram aferidas as temperaturas em cada um dos pontos nodais. Para as carnes mal 
passadas e ao ponto, o tempo total de estadia da amostra foi de 15 minutos, virando a carne após um intervalo de 10 minutos, para que houvesse cocção completa do exemplar. A carne bem passada foi assada por 20 minutos para que fosse possível atingir o grau de cocção desejado para essa modalidade, sendo que essa também foi virada após 10 minutos.

Salienta-se que a padronização das carnes como mal passadas, ao ponto e bem passadas foi realizada a partir da temperatura central da carne, aferida após a cocção. As carnes mal passadas possuem a temperatura interna central próxima a $55^{\circ} \mathrm{C}$, as ao ponto próxima de $63^{\circ} \mathrm{C}$ e as bem passadas próximas de $77{ }^{\circ} \mathrm{C}$. Em acréscimo, também foram analisados os aspectos visuais das carnes após a cocção. As carnes mal passadas apresentam aspecto rosado em todo o seu interior, as ao ponto possuem uma auréola rosada no centro da carne com o redor acinzentado e as bem passadas possuem aspecto acinzentado em sua extensão (RAMOS; GOMIDE, 2017).

$\mathrm{O}$ experimento foi replicado nove vezes, sendo 3 amostras para cada tipo de cocção (mal passada, ao ponto e bem passada). Ressalta-se, que independentemente do tipo de cocção obtido, a variação da temperatura com o tempo nas nove amostras durante os 15 primeiros minutos foi próxima, visto que as geometrias, os espaçamentos da malha e os equipamentos utilizados eram os mesmos. Ademais, essa hipótese é reforçada pelas baixas flutuações das temperaturas ambientais e do fluido acima da churrasqueira. Portanto, para fins matemáticos de cálculo das diferenças finitas, utilizar-se-á os noves pontos experimentais, maximizando a confiabilidade dos resultados. Acrescenta-se que o experimento foi realizado em dois dias, dessa forma, devido ao congelamento da carne, houve flutuações das temperaturas iniciais da amostra cárnea.

\subsection{Simulação}

Para execução de toda modelagem matemática, considera-se primeiramente, um pequeno elemento material de um corpo sólido, na forma de um paralelepípedo, de volume $\mathrm{d}_{\mathrm{x}} \mathrm{d}_{\mathrm{y}} \mathrm{d}_{\mathrm{z}}$, conforme mostrado na Figura 6.

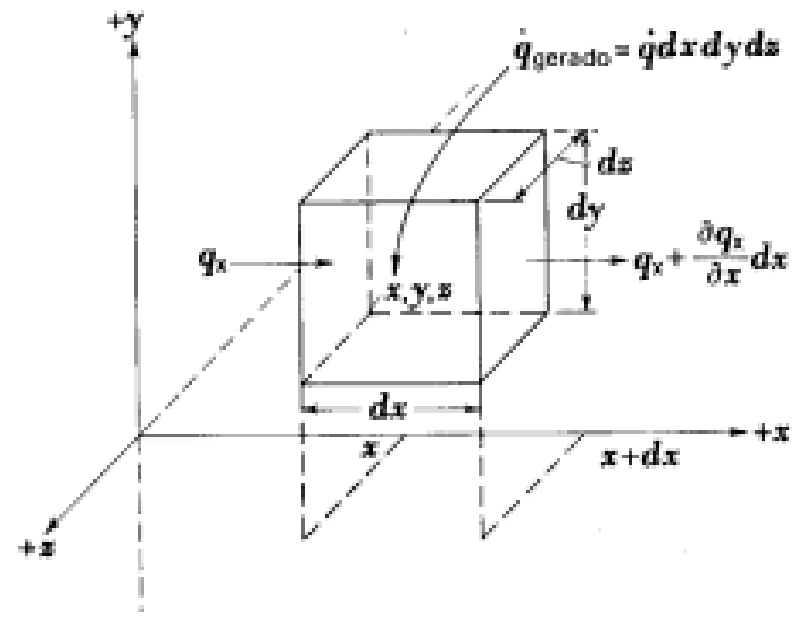

Figura 6 - Elemento material de corpo sólido, na forma de paralelepípedo, a partir do qual serão deduzidas as equações da transmissão de calor por condução para coordenadas cartesianas.

Fonte: Barrosa (2004).
O balanço de energia deste elemento pode ser calculado a partir da expressão:

$\begin{array}{cccc}\begin{array}{c}\text { Taxa de calor que } \\ \text { entra } \\ \text { no elemento por } \\ \text { unidade de tempo }\end{array} & +\begin{array}{c}\text { Taxa de calor } \\ \text { gerado no } \\ \text { elemento por } \\ \text { unidade de tempo }\end{array}=\begin{array}{c}\text { Taxa de calor que } \\ \text { sai } \\ \text { do elemento por } \\ \text { unidade de tempo }\end{array}\end{array}+\begin{gathered}\text { Taxa de variação } \\ \text { da energia } \\ \text { interna como } \\ \text { tempo }\end{gathered}$

Algebricamente, esta equação pode ser escrita como:

$$
\left(\dot{q}_{x}+\dot{q}_{y}+\dot{q}_{z}\right)+\frac{d \dot{q}}{d t}(d x \cdot d y \cdot d z)=\left(\dot{q}_{x+d x}+\dot{q}_{y+d y}+\dot{q}_{z+d z}\right)+\rho \cdot c \cdot \frac{\partial T}{\partial t}
$$

Em que $\frac{d \dot{q}}{d t}$ é a taxa de calor gerada por unidade de tempo e por unidade de volume, $T$ é a temperatura do corpo, $t$ é o tempo, $c$ é o calor específico do material e $\rho$ é a sua massa específica.

Segundo o cientista francês J. B. J. Fourier, a quantidade de calor transmitida por condução, segue a lei expressa por:

$$
q_{x}=-k \cdot A \cdot \frac{d T}{d x}
$$

Aplicando a Equação 5 a este problema, o calor transmitido por condução para dentro do corpo na direção x, mostrado na Figura 5 , pode ser descrito como:

$$
q_{x}=\left(-k \cdot \frac{\partial T}{\partial x}\right) d y \cdot d z
$$

Aplicando a mesma Equação 5 ao calor que deixa o corpo, por condução, na direção $\mathrm{x}$, pode-se escrever que:

$$
q_{x+d x}=\left[\left(-k \cdot \frac{\partial T}{\partial x}\right)+\frac{\partial}{\partial x}\left(-k \cdot \frac{\partial T}{\partial x}\right) d x\right] d_{y} \cdot d_{z}
$$

Fazendo a subtração dos dois termos, tem-se que:

$$
q_{x}-q_{x+d x}=\left[\frac{\partial}{\partial x}\left(k \cdot \frac{\partial T}{\partial x}\right)\right] d_{x} \cdot d_{y} \cdot d_{z}
$$

Aplicando de forma análoga a mesma Equação 5 para as direções y e z, pode-se chegar à Equação 1, anteriormente apresentada, que corresponde à equação geral para a transferência de calor por condução para coordenadas retangulares. Assumindo que as propriedades termo físicas $k, \rho$ e $c$ são constantes, a Equação 1 torna-se simplificada, e a nova equação, Equação 9, é conhecida então, como a Equação de Fourier.

$$
\frac{\partial T}{\partial t}=\frac{k}{\rho . c} \cdot\left(\frac{\partial^{2} T}{d x^{2}}+\frac{\partial^{2} T}{d y^{2}}\right)
$$


E nesse caso, $k$ representa a transferência de calor por condução, $\rho$ é a massa específica do material, $c$ é a capacidade calorífica do material, $T$ é a temperatura, $t$ é o tempo e $x$ é a posição espacial da carne.

\subsection{Condições inicial e de contorno}

A equação diferencial parcial (Equação 9) é conhecida com a equação do calor. Entretanto, tal solução depende das condições físicas existentes nas fronteiras do meio, e, se a situação muda ao longo do tempo, a solução também depende das condições existentes no sistema dado um instante inicial. Sendo a equação do calor de segunda ordem com relação às coordenadas espaciais, duas condições de contorno devem ser especificadas para dada coordenada necessária para descrever o sistema (INCROPERA; DEWITT, 2014).

A primeira condição de contorno que se fará uso, será a condição de Dirichlet, ou condição de contorno de primeira espécie, a qual admite a temperatura inicial do processo de cozimento da carne definido no tempo zero. Neste caso, vale deixar claro, que usamos esse tipo de condição porque a temperatura da superfície é constante e, que foi feita uma média dos valores iniciais da carne bovina utilizada. Assim:

$$
T_{\text {inicial }}=16,5^{\circ} \mathrm{C}
$$

Já para a outra condição de contorno, foi utilizada a condição de contorno de terceira espécie, justamente, pela existência de aquecimento por convecção na superfície e sua representação matemática pode ser obtida por balanço de energia no volume de controle. Dessa forma, tem-se que a transferência de calor por condução na carne, ao final do seu comprimento é igual a transferência de calor por convecção ao ambiente, matematicamente:

$$
\left.\frac{\partial T}{\partial z}\right|_{x=L}=-\frac{h}{k} \cdot\left(T_{x=L}-T_{\infty}\right)
$$

Onde $h$ é coeficiente de transferência de calor por convecção, $k$ é o coeficiente de transferência de calor por condução, $T_{x=L}$ é a temperatura ao final do comprimento da carne bovina e $T_{\infty}$ é a temperatura do fluido no ambiente.

Em relação ao método prático de medição das temperaturas das condições de contorno e do sistema como um todo, foi utilizado um termômetro digital de cozinha da marca Mary Tools cuja incerteza corresponde a $0,1^{\circ} \mathrm{C}$, justamente, por ser a menor leitura efetuada por esse dispositivo.

\subsection{Metodologia de resolução}

Uma vez que a rede nodal tenha sido estabelecida se faz necessário escrever a distribuição de temperatura para cada nó. Para que se realize a dedução das equações de diferenças finitas que descrevem as temperaturas em pontos distintos da rede nodal, é fundamental que se recorra ao balanço de energia.
Todas as deduções subsequentes levarão em consideração um sistema bidimensional em que $\Delta x$ é igual a $\Delta y$ estando esta consideração de acordo com a realização prática do experimento e adotar-se-á o método explícito de soluções, através das equações de diferenças finitas para cada ponto da rede nodal. Além disso, foi elaborado um croqui representativo (Figura 7) da geração de calor por parte da resistência térmica. Vale ressaltar, que a carne foi considerada uma superfície muito fina, e deste modo, haverá uma condução direta da taxa volumétrica de calor gerada pela churrasqueira.

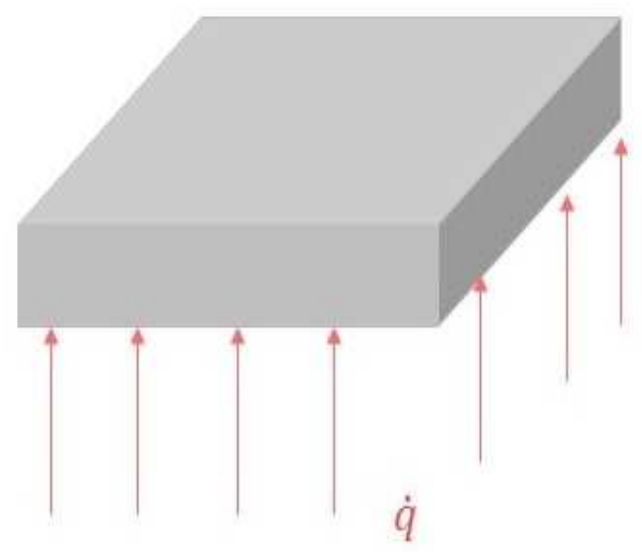

Figura 7 - Croqui representativo da taxa volumétrica de calor gerada através da resistência térmica.

Fonte: Elaborado pelo próprio autor.

\section{Nó interno}

Para um nó interno, representado pela Figura 8, tem-se a aplicação do balanço de energia.

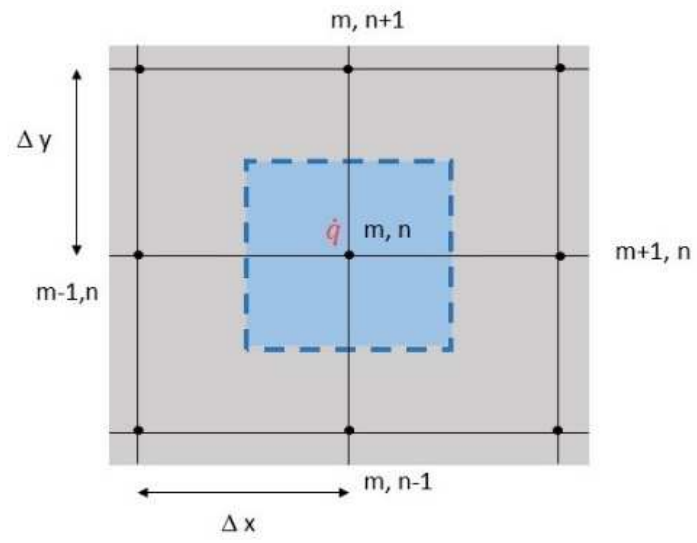

Figura 8 - Elemento material representativo, com nó interno, utilizado na dedução da equação das diferenças finitas pelo método explícito.

Fonte: Adaptado de Incropera e Dewitt (2014).

O balanço de energia para o nó interno com geração de energia pode ser escrito como:

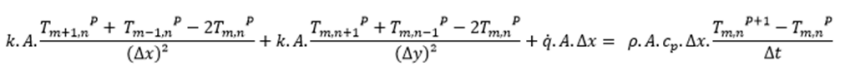


A equação que descreve o número de Fourier, pode ser escrita como:

$$
F_{O}=\frac{\alpha \cdot \Delta t}{(\Delta x)^{2}}
$$

Substituindo a Equações 11 na Equação 10 e rearranjando os termos, tem-se que o a temperatura de um ponto interior da rede nodal, quando $\Delta \mathrm{x}$ igual a $\Delta \mathrm{y}$, é descrita pela Equação 12:

$$
T_{m, n}{ }^{p+1}=F o\left(T_{m+1, n}{ }^{p}+T_{m-1, n}{ }^{p}+T_{m, n+1}{ }^{p}+T_{m, n-1}{ }^{p}+\frac{\dot{q} \cdot(\Delta x)^{2}}{k}\right)+(1-4 F o) T_{m, n}{ }^{p}
$$

O critério de estabilidade exigido pela equação de diferenças finitas para o nó interno é Fo $\leq 1 / 4$.

\section{Nó na superfície plana com convecção}

Para um nó na superfície plana com convecção, representado pela Figura 9, tem-se a aplicação do balanço de energia e encontra-se a Equação 13:

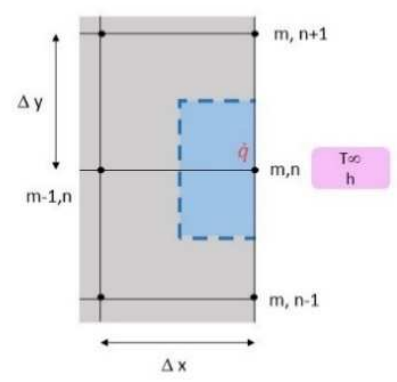

Figura 9 - Elemento material representativo, com nó na superfície plana com convecção, utilizado na dedução da equação das diferenças finitas pelo método explícito.

Fonte: Adaptado de Incropera e Dewitt (2014).

$$
\left(2 T_{m-1, n}{ }^{p}+T_{m, n+1}{ }^{p}+T_{m, n-1}{ }^{p}\right)-2 T_{m, n}{ }^{p}\left(2+\frac{h \cdot \Delta x}{k}\right)+\frac{2 \cdot h \cdot \Delta x T_{\infty}}{k}+\frac{2 \dot{q} \cdot \Delta x}{k}=\rho \cdot c \cdot \Delta x \cdot \frac{T_{m, n}^{p+1}-T_{m, n}{ }^{p}}{\Delta t}
$$
escrita como:

A equação que descreve o número de Biot pode ser

$$
B i=\frac{h \cdot \Delta x}{k}
$$

Substituindo as Equações 11 e 14 na Equação 13 e rearranjando os termos, tem-se que o a temperatura de um nó na superfície plana com convecção, quando $\Delta \mathrm{x}$ igual a $\Delta \mathrm{y}$, é descrita pela Equação 15:

$$
T_{m, n}^{p+1}=F o\left(2 T_{m-1, n}^{p}+T_{m, n+1}^{p}+T_{m, n-1}^{p}+2 B i T_{\infty}+\frac{\dot{q} \cdot(\Delta x)^{2}}{2 k}\right)+(1-4 F o-2 B i F o) T_{m, n}{ }^{p}
$$

O critério de estabilidade exigido pela equação de diferenças finitas para o nó na superfície plana com convecção é Fo $(2+\mathrm{Bi}) \leq 1 / 2$.

\section{Nó no vértice extremo com convecção}

Para um nó no vértice extremo com convecção, representado pela Figura 10, tem-se a aplicação do balanço de energia e encontra-se a Equação 16:

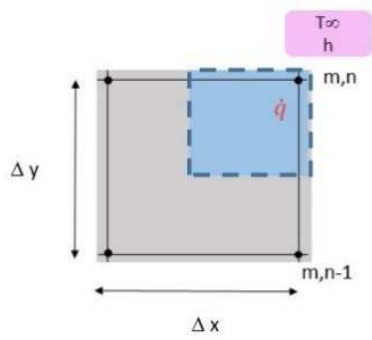

Figura 10 - Elemento material representativo, com nó no vértice extremo com convecção, utilizado na dedução da equação das diferenças finitas pelo método explícito.

Fonte: Adaptado de Incropera e Dewitt (2014).

$$
\left(T_{m-1, n}{ }^{p}+T_{m, n-1}{ }^{p}\right)-2 T_{m, n}{ }^{p}\left(1+\frac{h \cdot \Delta x}{k}\right)+\frac{2 \cdot h \cdot \Delta x T_{\infty}}{k}+\frac{4 \cdot \dot{q} \cdot \Delta x}{k}=\rho \cdot c \cdot \Delta x \cdot \frac{T_{m, n}^{p+1}-T_{m, n}{ }^{p}}{\Delta t}
$$

Substituindo as Equações 11 e 14 na Equação 16 e rearranjando os termos, tem-se que o a temperatura de um nó no vértice externo com convecção, quando $\Delta \mathrm{x}$ igual a $\Delta \mathrm{y}$, é descrita por:

$$
T_{m, n}{ }^{p+1}=2 F o\left(T_{m-1, n}{ }^{p}+T_{m, n-1}{ }^{p}+2 B i T_{\infty}+\frac{\dot{q} \cdot(\Delta x)^{2}}{4 k}\right)+(1-4 F o-4 B i F o) T_{m, n}{ }^{p}
$$

O critério de estabilidade exigido pela equação de diferenças finitas para o nó na superfície plana com convecção é Fo $(1+\mathrm{Bi}) \leq 1 / 4$.

\subsection{Parâmetros termo físicos}

Para a validação das equações das diferenças finitas do método explícito, se faz necessário calcular alguns parâmetros termo físicos referentes ao sistema, tais como: massa específica, coeficientes de transferência de calor por condução e convecção, comprimento característico, coeficiente de expansão térmica e viscosidade dinâmica. Para esse fim, utiliza-se grupamentos adimensionais, que exploram as consequências da homogeneidade dimensional das equações físicas.

Neste sentido, tem-se a Figura 11 representa o croqui da superfície utilizada no aquecimento juntamente com as áreas submetidas à convecção, inferindo que a correlação a ser utilizada é a referente à uma superfície inferior de placa aquecida. 


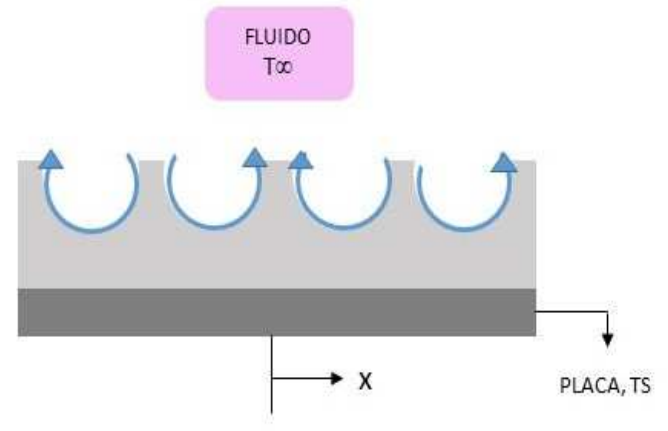

Figura 11 - Superfície inferior da placa (geometria adotada para o problema) submetida ao aquecimento e trocas de calor convectiva.

Fonte: Adaptado de Incropera e Dewitt (2014).

Dessa maneira, existe a necessidade de cálculo do número de Nusselt $(\mathrm{Nu})$, descrito pela Equação 2. O valor de L para o referente experimento é $0,0136 \mathrm{~m}$. Sequencialmente, tem-se a forma alternativa para se calcular o número de Rayleigh, na qual ele é relacionado com os números adimensionais de Grashof $\left(G r_{L}\right)$ e Prandtl $(P r)$. Dessa forma, tem-se para cada um dos grupamentos adimensionais:

\section{- PRANDTL}

A temperatura média do fluido foi de $86,4{ }^{\circ} \mathrm{C}$, o que equivale a $359,6 \mathrm{~K}$. Assim sendo, avaliou-se o número de Prandtl correspondente a partir da tabela A.4 de Incropera e Dewitt (2014). Como não havia um valor exato correspondente à temperatura em análise, efetuou-se uma interpolação simples, retornando o valor de Prandtl como 0,698.

\section{- $\quad$ GRASHOF}

Primeiramente, calcula-se o valor de $\beta$, sendo este $1 / T_{f}$ em que $T_{f}$ é a temperatura do fluido, correspondendo a um valor médio de 359,6 K, dessa forma $\beta$ é igual a $0,00278 \mathrm{~K}^{-1}$. O valor da viscosidade dinâmica foi avaliado por meio da tabela A.4 de. Incropera e Dewitt (2014). Como não havia um valor exato correspondente à temperatura em análise, efetuou-se uma interpolação simples, retornando o valor de $\mu$ como $212,410^{-7}$ (N.s $\left./ \mathrm{m}^{2}\right)$. Do mesmo modo, o valor de massa específica também foi interpolado utilizando a mesma referência e obteve-se $\rho$ igual a $0,9713 \mathrm{~kg} / \mathrm{m}^{3}$. O valor da gravidade adotado foi 9,81 $\mathrm{m} / \mathrm{s}^{2}$. A diferença de temperatura entre a carne e a superfície da churrasqueira $(\Delta T)$ foi estimado como sendo $183,5 \mathrm{~K}$, justamente porque a temperatura da churrasqueira foi avaliada como sendo $200{ }^{\circ} \mathrm{C}$ e a temperatura inicial média da carne é $16,5{ }^{\circ} \mathrm{C}$. Finalmente, expondo a formulação do número de Grashof, tem-se a Equação 18:

$$
G r=\frac{\rho^{2} g \cdot \beta \cdot \Delta T \cdot L^{3}}{\mu^{2}}
$$

Substituindo os valores numéricos:

$$
G r=\frac{\left.\left(0,9713 \frac{\mathrm{kg}}{\mathrm{m}^{3}}\right)^{2} \cdot 9,81 \mathrm{~ms}^{2} \cdot 0,00278 \cdot 1 / \mathrm{K} \cdot 183,5 \mathrm{~K} \cdot(0,0136)^{3}\right)}{\left(212,3 \times 10^{-7} \mathrm{~N} \cdot \frac{\mathrm{s}}{\mathrm{m}^{2}}\right)^{2}}
$$

- RAYLEIGH

O número de Rayleigh pode ser obtido através da Equação 19:

$$
R a_{L}=G r_{L} \cdot \operatorname{Pr}
$$

Substituindo os valores:

$$
\begin{gathered}
R a_{L}=262 \cdot 0,698 \\
R a_{L}=183,1
\end{gathered}
$$

Como $R a_{L}$ é um valor menor que $10^{4}$, admite-se, que por evidências das correlações utilizadas, o número de Nusselt é igual a 1. Desta maneira, rearranjando a Equação 2, chega-se à Equação 20 e torna-se possível calcular o coeficiente de de transferência de calor por convecção:

$$
h=N u \cdot \frac{k_{A r}}{L}
$$

O valor da condutividade térmica do ar foi avaliado por meio da tabela A.4 de Incropera e Dewitt (2014). Como não havia um valor exato correspondente à temperatura em análise, efetuou-se uma interpolação simples, retornando o valor de $k_{A r}$ como 30,7 x 10-3 (W/(mK)).

$$
\begin{gathered}
h=1 \cdot \frac{30,7 \cdot 10^{-2}\left(\frac{W}{m \cdot K}\right)}{0,0136 m} \\
h=22,6 \frac{W}{m^{2} \cdot K}
\end{gathered}
$$

Com o valor do coeficiente de película $(h)$, torna-se possível calcular o número de Biot, por meio da Equação 14, assim sendo:

$$
\begin{array}{r}
B i=\frac{22,6 \frac{W}{m^{2} \cdot K} \cdot 0,01 m}{30,7 \cdot 10^{-2}\left(\frac{W}{m \cdot K}\right)} \\
B i=0,7362
\end{array}
$$

Para efetuar o cálculo do número de Fourier, é necessário levar em consideração a maior restrição entre as equações, ou seja, adotando o critério de estabilidade exigido pela equação de diferenças finitas para o nó na superfície plana com convecção, isto é, Fo $(1+\mathrm{Bi}) \leq 1 / 4$. 


$$
\begin{gathered}
F o \leq \frac{1 / 4}{(1+B i)} \\
F o \leq \frac{1 / 4}{1+0,7362} \\
F o \leq 0,1440
\end{gathered}
$$

Para o cálculo do intervalo de tempo que se enquadra ao critério de estabilidade, utilizasse a própria definição do número de Fourier, descrita pela Equação 11:

$$
\Delta t=\frac{F o \cdot(\Delta x)^{2}}{\alpha}
$$

O valor da difusividade térmica do ar foi avaliado por meio da tabela A.4 de Incropera e Dewitt (2014). Como não havia um valor exato correspondente à temperatura em análise, efetuou-se uma interpolação simples, retornando o valor de $\alpha$ como $31,510^{-6}\left(\mathrm{~m}^{2} / \mathrm{s}\right)$.

$$
\begin{gathered}
\Delta t=\frac{0,1440 \cdot(0,01 \mathrm{~m})^{2}}{\frac{31,5 \cdot 10^{-6} \mathrm{~m}^{2}}{\mathrm{~s}}} \\
\Delta t=0,46 \mathrm{~s}
\end{gathered}
$$

Para estar bem situado no limite de estabilidade, optase por $\Delta t=0,4 \mathrm{~s}$, que corresponde a:

$$
F o=\frac{\frac{31,5 \cdot 10^{-6} m^{2}}{s} \cdot(0,4 s)}{(0,01 m)^{2}}
$$

Agora, se faz necessário avaliar o coeficiente médio de condução da carne. Para isso, utilizou-se a Equação 21, que representa uma correlação descrita por Dominguez (1974) citado por Rao (1986) para que se possa calcular o $k$ da carne bovina.

$$
k=0,6 X_{W}+0,2 X_{p}+0,245 X_{C}+0,18 X_{f}
$$

Onde $X_{W}$ é a fração de água, $X_{p}$ é a fração de proteína, $X_{C}$ é a fração de carboidrato e $X_{f}$ é a fração de gordura na carne. Considerando a Tabela 1, na qual é descrita a composição da carne bovina, tem-se:

Tabela 1 - Composição da carne bovina "coxão mole"

\begin{tabular}{cc}
\hline \multicolumn{2}{c}{ CARNE BOVINA } \\
\hline Composição & Porcentagem relativa \\
\hline Água & 0,75 \\
\hline Proteína & 0,23 \\
\hline Gordura & 0,20 \\
\hline
\end{tabular}

Fonte SEU $\beta, 1991$
Substituindo os dados da Tabela 1 na Equação 21, calcula-se o valor da condutividade térmica da carne. Dessa maneira:

$$
\begin{gathered}
k=0,6 \cdot 0,75+0,2 \cdot 0,23+0,245 \cdot 0+0,18 \cdot 0,02 \\
k=0,4996 \frac{w}{m \cdot K}
\end{gathered}
$$

Por fim, para se determinar o tempo em que a carne ficará pronta de acordo com os estágios de preparo avaliados, se faz necessário medir a temperatura no centro da carne. De acordo com Ramos e Gomide (2017), as carnes mal passadas possuem a temperatura interna central próxima a $55^{\circ} \mathrm{C}$, as ao ponto próxima de $63^{\circ} \mathrm{C}$ e as bem passadas próximas de $77^{\circ} \mathrm{C}$.

\section{RESULTADOS E DISCUSSÃO}

Os dados de temperatura referentes ao experimento de aquecimento da carne bovina foram recolhidos com o auxílio de um termômetro de cozinha, durante intervalos de tempo préestabelecidos: $0,5,10,15$ minutos, e, no caso da carne bem passada houve a retirada dos dados no tempo referente a 20 minutos. Os resultados práticos estão apresentados nas Figuras 11,12 e 13 .

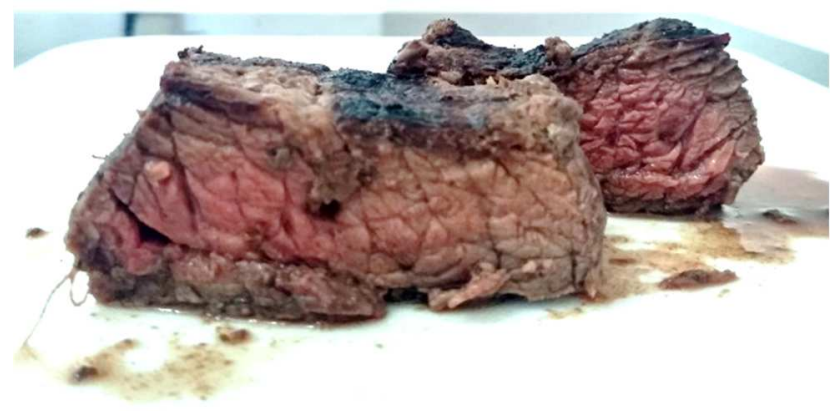

Figura 12 - Carne mal passada, temperatura média no centro igual a $53,9^{\circ} \mathrm{C}$.

Fonte: Autores.

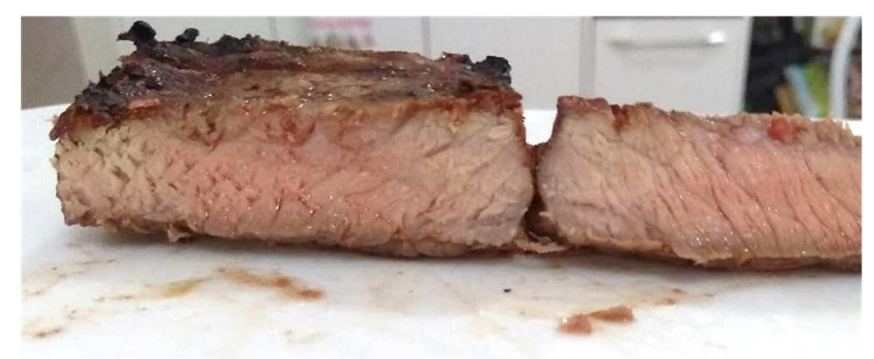

Figura 13 - Carne ao ponto, temperatura média no centro igual a $65,4^{\circ} \mathrm{C}$.

Fonte: Autores. 


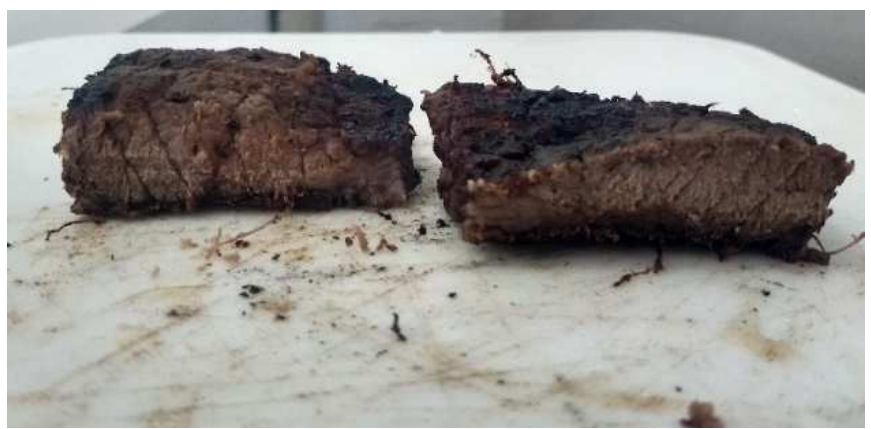

Figura 14 - Carne bem passada, temperatura média no centro igual a $76,7^{\circ} \mathrm{C}$. Fonte: Autores.

Todos os dados de temperaturas e tempo foram compilados em uma planilha do Excel para que pudessem receber um tratamento matemático adequado. Nesse sentido, foi determinado que os cálculos referentes às equações de diferenças finitas pelo método explícito seriam tratadas até o tempo total de 15 minutos, justamente por representar um espaço amostral maior - 9 amostras mais precisamente $-\mathrm{e}$ isso se deve ao fato de que, até esse tempo de aquecimento, todas as amostras de carne bovina estariam submetidas às mesmas condições.

Como o espaçamento de tempo, resultante da fórmula do Fourier que convergia foi de 0,4 segundos, tem-se que o número de pontos gerados foi muito elevado, visto que:

$$
\begin{gathered}
N . \text { de pontos }=\frac{15 \mathrm{~min} x \frac{60 \mathrm{~s}}{1 \mathrm{~min}}}{0,4 \mathrm{~s}} \\
\text { N.de pontos }=2.250
\end{gathered}
$$

Esse valor corresponde a cada um dos pontos da malha, ou seja, de 1 até 30, como representado na Figura 15.

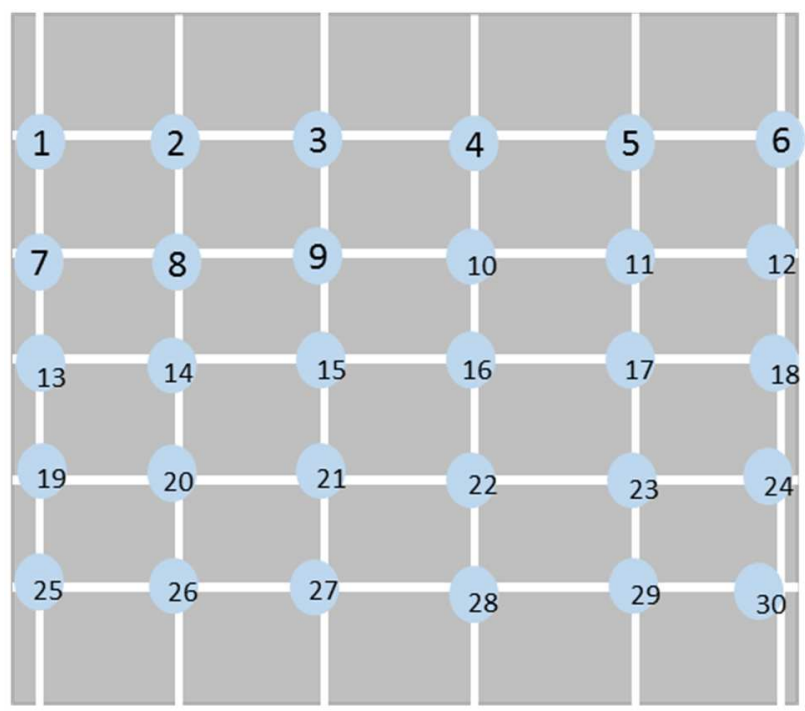

Figura 15 - Croqui representativo da denominação e localização dos pontos da malha.

Fonte: Autores.
Para que o código de toda programação da planilha do Excel pudesse ser elaborado foi utilizado um esboço, o qual se encontra na Figura 16 com o intuito de realizar as deduções das equações do método das diferenças finitas para cada um dos pontos. Ressalta-se que foi criado um eixo de simetria imaginário na malha, possibilitando a equivalência dos valores de temperaturas em pontos específicos da malha.

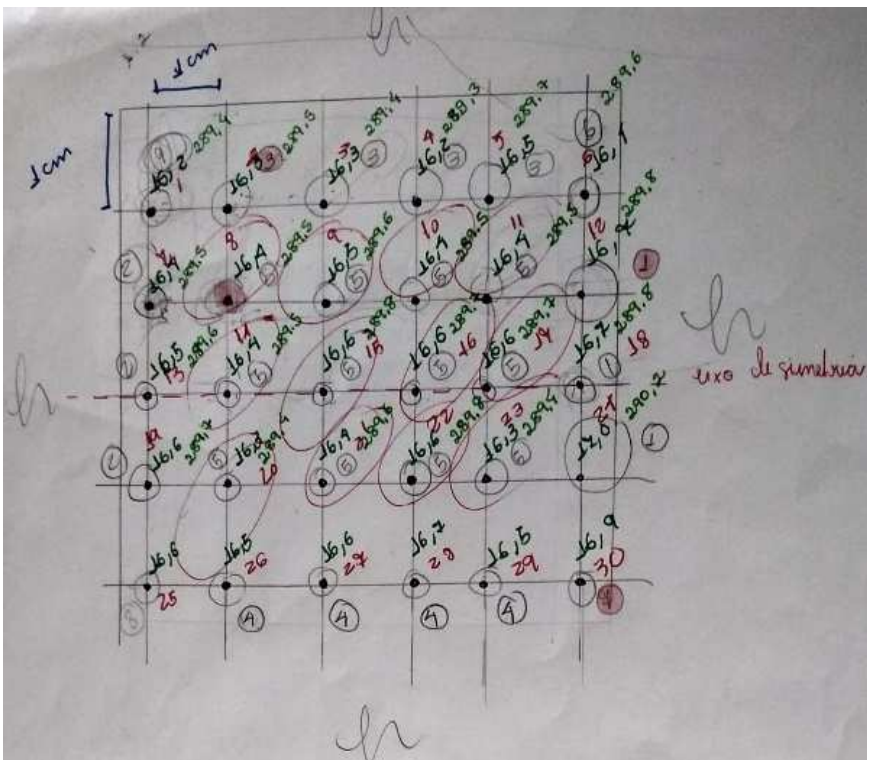

Figura 16 - Esboço da malha nodal utilizada nos cálculos.

Fonte: Autores.

A partir da projeção dos valores, Figura 17, fica evidente que, por este cálculo, a carne em um intervalo de tempo equivalente a 300 segundos, atingiria valores de temperatura por volta dos $90^{\circ} \mathrm{C}$. É notável que os valores convergiram depois de um intervalo de tempo muito grande, o que está de acordo com as predições do método.

Com o intuito de analisar a dispersão dos valores experimentais e calculados, foi plotado um segundo gráfico, Figura 18, em que foram utilizados os dados correspondentes a até 900 segundos (15 minutos), pois este é o tempo limite, sob o qual tem-se informações experimentais para a análise. 


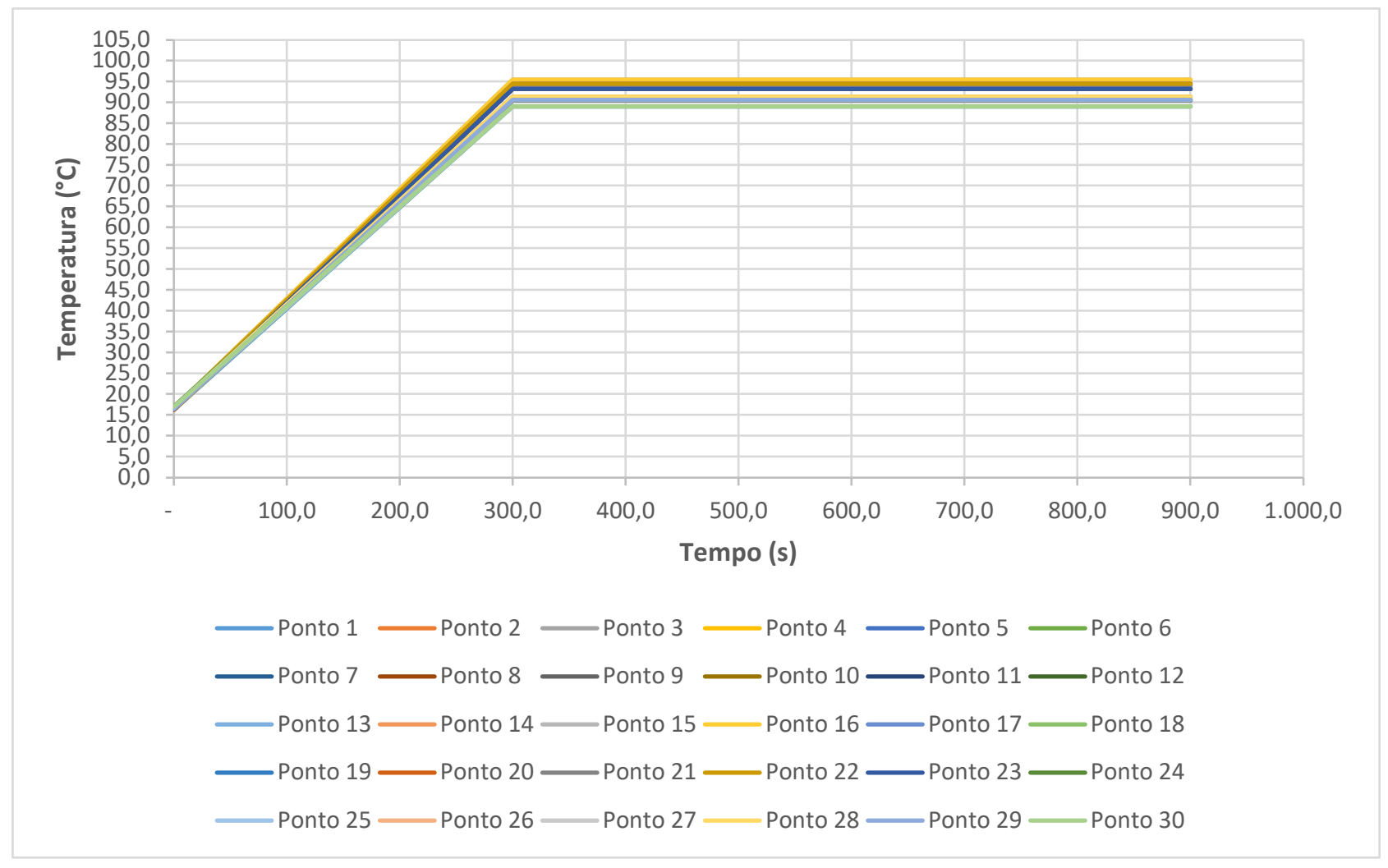

Figura 17 - Gráfico gerado a partir do cálculo por diferenças finitas pelo método explícito com valores de tempo sintetizados.

Fonte: Autores.

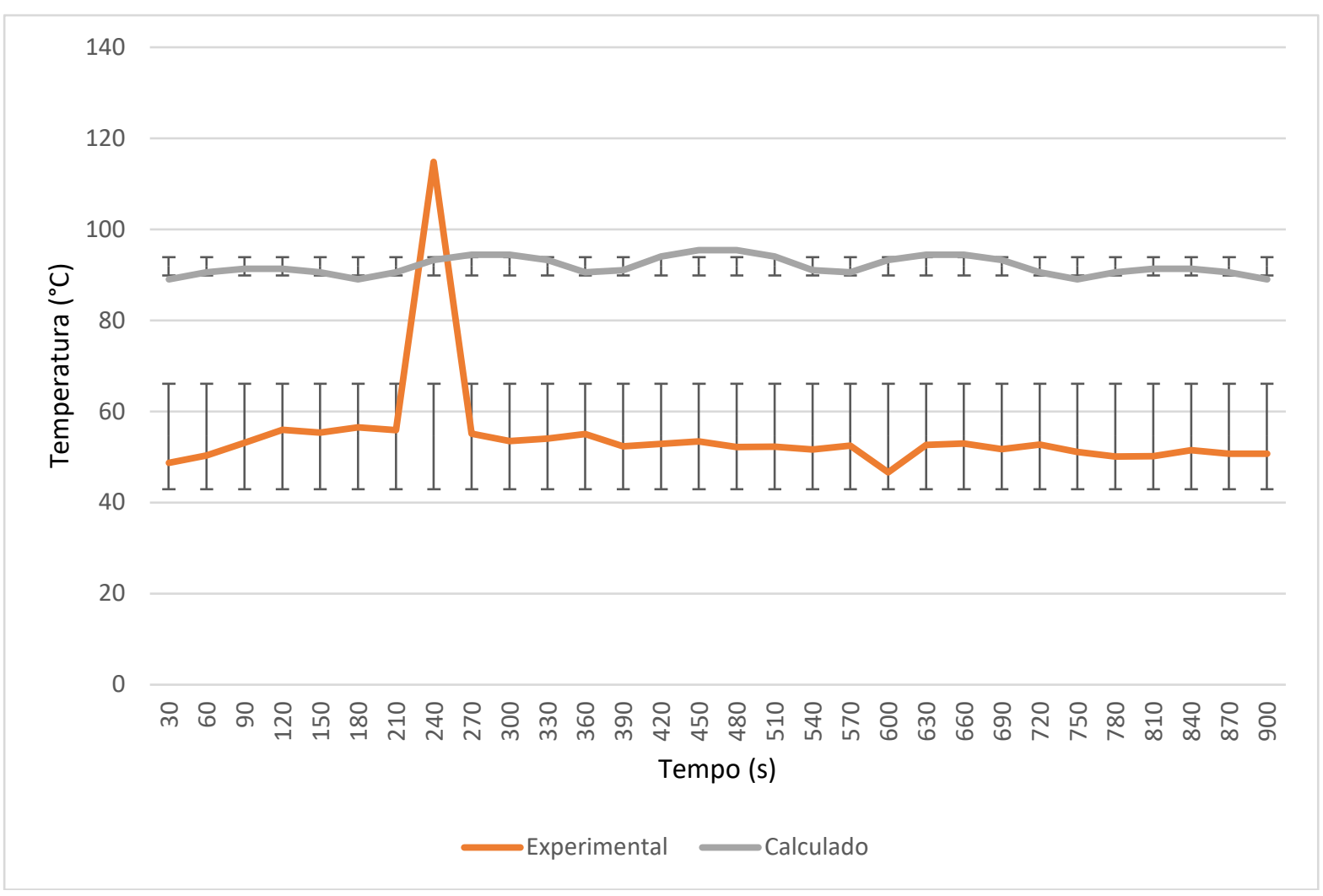

Figura 18 - Gráfico gerado a partir da variância entre os valores experimentais e calculados. Fonte: Autores. 
Os dados mostram que a variância dos valores calculados foi bem reduzida, quando comparadas àquela dos valores experimentais, sendo o ponto 8 , com uma temperatura de $114,9{ }^{\circ} \mathrm{C}$, o ponto com maior variância de todo o conjunto.

Os erros certamente surgiram pelo fato da geração volumétrica não corresponder de forma efetiva ao valor adotado, $\dot{q}=1,67 \cdot 10^{7} \mathrm{~W} / \mathrm{m}^{3}$, justamente por haver perdas de calor para o ambiente e também pelo fato da churrasqueira Grill utilizada não apresentar um rendimento de $100 \%$ e não ser capaz de direcionar todo o seu fluxo térmico para a amostra de carne utilizada.

Além disso, para que os cálculos pudessem ser realizados, a amostra foi tratada como uma placa fina e retangular, o que evidentemente seria o mais adequado, pois a carne utilizada apresentava cerca de $2,5 \mathrm{~cm}$ de altura e dispunha de irregularidades ao longo de seu formato.

Outro fator que pode ter contribuído para a geração dos erros foi $o$ fato das correntes convectivas apresentarem variações e nos cálculos foi considerado apenas uma média das medidas experimentais. E, por fim, tem-se a influência da falta de estabilidade do termômetro utilizado, o que interfere diretamente nas temperaturas lidas durante o experimento.

Ademais, acrescenta-se que todos os valores apresentam um valor de incerteza associado à incerteza do termômetro de $\pm 0,1{ }^{\circ} \mathrm{C}$ (menor medida aferida pelo termômetro digital) e à incerteza da régua, utilizada para medir as frações de carne, de $\pm 0,0005 \mathrm{~m}$ (metade da menor leitura possível da régua graduada). Dessa forma, as incertezas foram propagadas ao longo do procedimento experimental e para determiná-las foi utilizada a Metodologia de Kline e McClintock, conforme discutido e apresentado em Andrade et al. (2017). Portanto, as Equações 12, 15 e 17 foram derivadas em relação ao tempo e em relação à variação $\Delta \mathrm{x}$. Essas derivadas foram substituídas na Equação 22 e multiplicadas pela incerteza do termômetro e da régua.

$$
W R=\sqrt{\left[\left(\frac{d R}{d x 1} w 1\right)^{2}+\left(\frac{d R}{d x 2} w 2\right)^{2}\right]}
$$

Na Equação 22 o termo WR representa a incerteza obtidas considerando a propagação de incertezas; $\frac{d R}{d x 1}$ representa a derivação da equação das diferenças finitas em relação às temperaturas e termo w1 representa a incerteza associada ao termômetro. Analogamente, $\frac{d R}{d x 2}$ é a derivação da equação de diferenças finitas em relação a $\Delta \mathrm{x}$ e w2 representa a incerteza da régua.

Ressalta-se que pontos com a mesma formulação apresentavam o mesmo valor de incerteza, assim foram obtidos os valores de $\mathrm{WR}= \pm 0,108{ }^{\circ} \mathrm{C}$ para os nós internos, de $\mathrm{WR}= \pm$ $0,102{ }^{\circ} \mathrm{C}$ para os nós na superfície plana com convecção e WR $= \pm 0,069{ }^{\circ} \mathrm{C}$ para os nós no vértice extremo com convecção. Em acréscimo, salienta-se que essas incertezas podem ser arredondadas para o valor de $0,1^{\circ} \mathrm{C}$ para atender à quantidade de algarismos significativos das medidas aferidas.

\section{CONSIDERAÇÕES FINAIS}

O objetivo do estudo foi atingido, tendo em vista que através das medidas experimentais foi possível observar o tempo de preparo para os diferentes pontos da carne bovina "coxão mole". Os conceitos de transferência de calor que se referem aos processos de condução e convecção puderam ser evidenciados na prática, bem como a metodologia de cálculo por equações de Diferenças Finitas pelo Método Explícito.

Os erros do processo foram elevados, justamente porque as considerações adotadas para a modelagem do problema não foram totalmente adequadas, conforme evidenciado anteriormente. Inicialmente, pontua-se que que para a aferição das temperaturas na malha nodal era requerido um intervalo de tempo de aproximadamente 4,5 minutos. Dessa forma, as temperaturas associadas a cada intervalo de tempo, na prática foram lidas em tempos diferentes.

Destaca-se, também, a ocorrência de um erro experimental devido ao posicionamento da amostra de carne, visto que essa foi disposta de maneira errônea na churrasqueira, sendo realizada a averiguação de temperatura em pontos que, em teoria, não deveriam apresentar variações nesse parâmetro. Adiciona-se a isso, o fato do termômetro requerer um intervalo de tempo para entrar em equilíbrio térmico com a amostra, o que gerou medidas pouco precisas.

Em acréscimo, tem-se que as correntes convectivas do local de realização do experimento podem ter afetado nos resultados, tendo em vista que as temperaturas do fluido alteravam-se a cada amostra. Ademais, tem-se que a consideração de que a fração de carne é próxima a uma superfície delgada caracteriza-se errônea, justamente porque ela possuía uma altura equivalente a $2,5 \mathrm{~cm}$. Soma-se a isso, o fato de a carne não ter composição homogênea, visto que apresenta gorduras e fibras desigualmente distribuídas por sua extensão, afetando a regularidade da amostra e, consequentemente, alterando o coeficiente de condutividade térmica.

Tem-se ainda que apesar dos resultados obtidos não serem totalmente coerentes com a realidade, o trabalho evidenciou a importância de se levar em consideração propriedades termofísicas adequadas e não somente aspectos visuais dos produtos. Ressalta-se, a importância crucial dos fenômenos de transferência de calor na indústria alimentícia para controle biológico de patogênicos que afetam a saúde dos serem humanos, como Listeria monocytogenes, Salmonella e Escherichia coli.

Por fim, o Método da Diferenças Finitas pode ser aplicado à gastronomia, possibilitando que as corporações de restaurantes possam determinar o estado de cocção dos seus pratos por meio de previsões de temperatura. 
Os autores agradecem o apoio por parte da Universidade Federal de Viçosa (UFV). O presente trabalho foi realizado com apoio da Coordenação de Aperfeiçoamento de Pessoal de Nível Superior - Brasil (CAPES) - Código de Financiamento 001 e da Fundação de Amparo à Pesquisa do Estado de Minas Gerais (FAPEMIG).

\section{R E F E R E N C I A S}

ANDRADE, A. C. A.; REIS, H. F. A. F.; SIQUEIRA, A. M. de O.; MADUREIRA, M. F.; GOUVEA, N. A.; GONZAGA, L. F.; MENEZES, T. L. de; FREIRE, B. H. de F. PROPAGAÇÃO DE INCERTEZAS: UM EXPERIMENTO ACADÊMICO SIMPLES. The Journal of Engineering and Exact Sciences, [S. 1.], v. 3, n. 3, p. 358-368, 2017. DOI: 10.18540/jcecvl3iss3pp358$368 . \quad$ Disponível em: https://periodicos.ufv.br/jcec/article/view/24469416030 32017358. Acesso em: 19 fev. 2020.

BARROSA, M. Princípios fundamentais da transferência de calor. 2004. Disponível em: < http://sites.poli.usp.br/p/jesse.rebello/termo/Trabalho_T ranscal.pdf $>$. Acesso em: 19 nov. 2020.

BEJAN, A. Transferência de Calor, Ed. Edgard Blucher Ltda., 1996.

ÇENGEL, Y.; GHAJAR, A. Transferência de Calor e Massa - uma abordagem prática. Tradução de Fátima A. M. Lino, $4^{\mathrm{a}}$ ed. AMGH Editora Ltda.: Porto Alegre, 2012.

DERMACHI, O. S.; SILVA, M. A. M. R.; FIORENTINO, P. L. Churrasco, elétrico ou carvão? 2012. FEEC/ UNICAMP.

DOMINGUEZ, D.; RAO, R. (1974) citado por Rao e Rizvi, Tabela de B.4 Equações para o cálculo de condutividade térmica de alimentos, 1986.

FRANÇA, F.C.O.; MENDES, A.C.R.; ANDRADE, I.S.; RIBEIRO, G.S.; PINHEIRO, I. B. Mudanças dos hábitos alimentares provocados pela industrialização e o impacto sobre a saúde do brasileiro. Primeiro seminário de alimentação e cultura na Bahia. 2012.

INCROPERA, F.; DEWITT, D.P. Fundamentos de Transferência de Calor e de Massa, $7^{\mathrm{a}}$ ed., Rio de Janeiro: LTC, 2014.

PACÍFICO, A. Análise Dimensional. 2017. Disponível em: $<$ https://edisciplinas.usp.br/pluginfile.php/2889753/mod _resource/content/5/2.1-

analise_dimensional_e_semelhanca.pdf $>$. Acesso em: 23 nov. 2019.

RAMOS, E.; GOMIDE L. Avaliação da qualidade de carnes - fundamentos e metodologias. 2. Ed. Ver. E ampl. Viçosa, MG: Ed. UFV, 2017.

SEU $\beta$, I. Valor nutricional de la carne y de los productos cárnicos. Consideraciones críticas sobre sus componentes en comparación con otros alimentos. Fleischwirtsch, español, n.1, p.47-50, 1991. 